\begin{tabular}{|l|l|l|l|l|}
\hline Revista Clío América & ISSN: 1909-941X & Vol. 8 & No. 16 & Julio - Diciembre de 2014 \\
\hline
\end{tabular}

\title{
La PYME como generadora de empleo en México
}

\author{
SME as a job in Mexico
}

María Luisa Saavedra García Doctora en Administración Universidad Nacional Autónoma de México, México maluisasaavedra@yahoo.com

Miriam Edith Saavedra García Maestra en Administración Universidad del Mar, Huatulco, Oaxaca, México mirisaga@hotmail.com

Tipología:

Artículo de Investigación Científica y Tecnológica

Fecha de Recibido: Mayo 30 de 2014

Fecha de Aceptación: Julio 15 de 2014

Para citar este artículo:

Saavedra, G. M., \& Saavedra, G. M. (2014). La pyme como generadora de empleo en México, Clío América, 8 (16), 153 - 172
Resumen: El objetivo de este trabajo consiste en mostrar la importancia de la PYME en México como fuente generadora de empleos, para este fin en primer lugar se desarrollan los temas de las políticas de generación empleo y las PYME y las políticas empresariales; se trata de un trabajo de tipo documental con una recolección de datos que permitieron analizar la evolución del sector empresarial mexicano por los años 1994 a 2008.

Los principales hallazgos permiten determinar que las PYME sobre todo las micro empresas son un importante generador de empleos en México. Sin embargo, es de notar que ha ido perdiendo participación frente a la empresa grande sobre todo en el sector manufacturero.

Palabras clave: PYME, Empleos, Políticas.

JEL: J21, J82, L23, L53

Abstract: The aim of this paper is to show the importance of SMEs in Mexico as a source of jobs, for this purpose first issues of employment generation policies and SMEs and corporate policies are developed; It is a documentary work with a collection of data that allowed analyzing the evolution of Mexican business sector for the years 1994-2008.

The main findings can determine that SMEs especially micro businesses are an important source of jobs in Mexico. More however, it is noteworthy that it has been losing share to big business especially in the manufacturing sector.

Keywords: SME, Jobs, Policy. 


\section{Introducción}

La micro pequeña y mediana empresa (PYME) es un sector de mucha importancia tanto en México como en el mundo, en Latinoamérica, alcanza en promedio el $99.25 \%$ del total de las unidades empresariales generando el $35.5 \%$ del empleo con lo que cumple así un importante papel de niveladora de la desigualdad económica, que caracteriza a esta región (Saavedra y Hernández, 2008). En Europa el $99 \%$ de las empresas son PYME (CE, 2006), en Estados Unidos lo son el $99.7 \%$ del total de las empresas (Leebaert, 2006).

Su importancia también radica en que las pequeñas empresas pueden ayudar a reducir la pobreza en los países, puesto que generan gran cantidad de empleo, niveles adecuados de calidad de empleo y bajo costo de bienes y servicios utilizados por los pobres (Vanderberg, 2007).

El aporte de las PYME en la generación de empleos lo podemos ver en la siguiente tabla:

-Tabla 1.

Importancia de la PYME en la producción y el empleo (Varios años)

\begin{tabular}{|l|c|c|}
\hline \multicolumn{1}{c|}{ País } & Participación en el empleo formal & Participación en la producción \\
\hline Argentina & $70.2 \%$ & $53.7 \%$ \\
\hline Brasil & $59.8 \%$ & $34.3 \%$ \\
\hline Chile & $63.0 \%$ & $20.4 \%$ \\
\hline Colombia & $67.2 \%$ & $38.7 \%$ \\
\hline Ecuador & $55.0 \%$ & $20.0 \%$ \\
\hline México & $75.0 \%$ & $62.0 \%$ \\
\hline Paraguay & $77.0 \%$ & - \\
\hline Perú & $67.9 \%$ & $55.5 \%$ \\
\hline Uruguay & $68.5 \%$ & - \\
\hline Venezuela & $38.1 \%$ & - \\
\hline Argentina-CNE 2004 & $66.3 \%$ & $50.2 \%$ \\
\hline Unión & $67.1 \%$ & $57.6 \%$ \\
\hline Europea-27-2005 & &
\end{tabular}

Fuente: Cohen y Baralla (2012). 
Como podemos ver en la tabla 1 el aporte de las PYME en la generación de empleos es en promedio del $60 \%$, para los países analizados y la participación en la producción alcanza 40\%. Sin embargo, no son ajenas a las amenazas del entorno que en determinado momento pueden limitar su desempeño (Benacek, 1995), por lo que resulta necesario que los países generen políticas que las protegan.

De ahí la importancia estratégica que éstas adquieren o deberían tener en términos del desarrollo económico nacional, regional o local, en México, en el año 2009 se contaba con más de 5 millones de empresas (incluyendo a productores agrícolas, ganaderos, acuicultores, mineros, artesanos, manufacturas, comercios, servicios turísticos y culturales) (Saavedra y Tapia, 2012), en la tabla 2 se puede ver la distribución de estas empresas de acuerdo con el tamaño:

Tabla 1.

\section{Distribución de empresas mexicanas}

\begin{tabular}{|l|c|c|c|c|}
\hline Empresas & $\begin{array}{c}\mathrm{N}^{0} \text { de } \\
\text { empresas }\end{array}$ & $\begin{array}{c}\% \text { del total de } \\
\text { empresas }\end{array}$ & $\begin{array}{r}\% \text { de generación de } \\
\text { empleos }\end{array}$ & $\begin{array}{c}\% \text { de aportación al } \\
\text { PIB }\end{array}$ \\
\hline Micro & $4,877,070$ & $94.80 \%$ & $45.60 \%$ & $15.50 \%$ \\
\hline Pequeñas & 214,956 & $4.18 \%$ & $23.80 \%$ & $14.50 \%$ \\
\hline Medianas & 42,415 & $0.82 \%$ & $9.10 \%$ & $22.00 \%$ \\
\hline PYME & $5,134,441$ & $99.80 \%$ & $78.5 \%$ & $52.0 \%$ \\
\hline Grandes & 9,615 & $0.20 \%$ & $21.50 \%$ & $48.00 \%$ \\
\hline Total & $5^{\prime} 144,056$ & $100.00 \%$ & $100.00 \%$ & $100.00 \%$ \\
\hline
\end{tabular}

Fuente: Elaboración propia con base en Censos Económicos INEGI (2010).

Como se puede apreciar en la tabla 2, la importancia de las PYME en México es relevante si se considera que representan el $99.80 \%$ del total de las unidades económicas, generan el $78.5 \%$ del empleo y aportan el $52 \%$ al Producto Interno Bruto (PIB) nacional.

Por ello, el objetivo de este trabajo consiste en mostrar de qué manera la PYME es un importante motor para la generación de empleos en México, por lo que se desarrolla el tema de las políticas de generación de empleo y las PYME, así como el tema de políticas empresariales, realizando enseguida un análisis de la evolución de este sector, mostrando por ultimo las conclusiones.

\section{Las Políticas de generación de empleo y las PYME}

Existen numerosos estudios acerca de la influencia de las políticas públicas en el desarrollo de las PYME (Bibu, Stefea \& Sala, 2009, Carree, Van, Thurik, \& Wennekers, 2002, Carroll, Holtz-Eakin, Rider, \& Rosen, 2000, Choi y Phan, 2006, Cohen y Baralla, 2012, Di Tomaso y Dubbini, 2000, Dussel, 2004, Ramírez, 2007, Rivera, 2002, Roper, 2005, Smallbone y Welter, 2001, Zevallos, 2006), todos coinciden en la importancia del papel del gobierno para impulsar el desarrollo de estas empresas. De otro lado, las políticas que pretenden reducir la pobreza a través 
de la creación de empleos nuevos ponen especial atención en las PYME, mejorando su acceso al financiamiento, y el medio ambiente de negocios así como las condiciones generales de mercado, estas pueden tener un efecto positivo sobre el número de empleos creados por las PYME, especialmente en el largo plazo (De Kok, Deijl \& Veldhuis-Van, 2013).

De acuerdo con el Banco Mundial (2013), 200 millones de personas en el mundo carecen de empleo y una gran parte de estas se encuentran en situación de pobreza. Por consiguiente, una estrategia importante para combatir la pobreza consiste en crear nuevos empleos, aquí es donde juegan un papel fundamental las PYME. Esto se corrobora con los estudios de Ayyagari et al. (2011) quienes encontraron en un estudio longitudinal de los años 2006 a 2010 de 99 países, que las PYME generan más empleo que las empresas grandes. Pero lo anterior no es reciente, Birch (1979 citado por Kliesen y Maués, 2011), ya había comprobado con un estudio empírico que las pequeñas empresas (con menos de 20 empleados) generaron dos tercios de los nuevos empleos entre 1969 y 1976, y las empresas con menos de 100 empleados generaron el $85 \%$ de nuevos empleos mientras que las empresas grandes (500 o más empleados) solo generaron el 15\% de los nuevos empleos.

Por su parte, Haltiwanger, Jarmin y Miranda (2012) también encontraron que la tasa de crecimiento de generación de empleos es más alta en las empresas pequeñas que en las grandes, sin embargo comprobaron que las empresas jóvenes tienen alta capacidad de generación como de destrucción de empleos. Así también, Neumark, Wall \& Zhang (2008) encontraron que la relación negativa entre el tamaño del establecimiento y la creación de empleo es mucho menos clara para el sector manufacturero, es decir no encontraron una mayor tasa de crecimiento del empleo en las pequeñas empresas del sector manufacturero comparado con las empresas grandes.

Miliaras (2012) señala que aunque en Estados Unidos se han encontrado numerosos estudios que demuestran que la pequeña empresa genera más empleo que la grande, en los países en vías de desarrollo existe poca evidencia de que eso sea así. De otro lado, Abedul (citado por Miliaras, 2012) encontró en un estudio realizado en los años setentas en Estados Unidos, que las empresas Gacela eran las que generaban un mayor número de empleos (Abedul definió a las Gacela como pequeñas empresas que mostraban un crecimiento en ventas superior a $20 \%$ anual en un periodo de cuatro años). El estudio concluye señalando que las empresas Gacela son las que deberían tener más atención por parte de las políticas públicas.

Promover el crecimiento del empleo, a través de políticas que favorezcan a las pequeñas empresas pueden tener ventajas y desventajas, De este modo tenemos que (CBO, 2012):

- Una ventaja está relacionada con el costo de cumplir con las regulaciones federales, que puede ser especialmente gravoso para las pequeñas empresas. Por ejemplo, el cumplimiento de leyes federales, tales como las destinadas a evitar la discriminación 0 a reducir la contaminación a menudo requiere que las empresas desarrollen sistemas o procedimientos internos que tienen un costo fijo por empresa, además de los costos que varían con el tamaño de la firma. En ese caso, la consecución del objetivo de la regulación en pequeñas empresas será relativamente más costoso de lograr que en las empresas grandes. Por lo tanto, las autoridades pueden razonablemente decidir la aplicación de ciertas normas modificadas para las pequeñas empresas 0 eximirlas de algunas regulaciones. Este enfoque podría promover el crecimiento del empleo.

- En contraste, una desventaja de las políticas que favorecen a las pequeñas empresas es que tales políticas pueden inadvertidamente desalentar a las empresas para aumentar en tamaño y perder este tratamiento preferente. Por otra parte, eximir a las pequeñas empresas a partir de ciertos reglamentos 0 normas modificatorias aplicados a las mismas, puede permitir que persistan algunos problemas, tales como discriminación 0 contaminación.

Asimismo, se ha analizado que se puede estimular el empleo reduciendo temporalmente los impuestos patronales sobre la nómina cuando las pequeñas empresas contratan trabajadores adicionales. Sin embargo, dado que los trabajos proporcionados 
por las empresas pequeñas son menos "durables" (es decir, que los puestos de trabajo son más propensos a ser eliminados más tarde), el beneficio económico de cada puesto de trabajo subvencionado es menor si el impuesto reducido a la nómina se limita a las pequeñas empresas. Además, debido a una mayor volatilidad del empleo en las pequeñas empresas, una buena parte de la reducción de los impuestos destinados a las pequeñas empresas sería como pagar por el crecimiento del empleo que se habría producido sin la política, lo que reduce el costo-efectividad de la política relativa a la reducción de impuestos.

\section{Características de las empresas y su relación con el crecimiento del empleo}

Estudios recientes han identificado varias características propias de las empresas que están relacionadas con la tasa de crecimiento del empleo (Tanto para PYME como para empresas grandes). Como podemos ver en la siguiente tabla:

|Tabla 3.

Relación entre las Características de las empresas y tasa de crecimiento del empleo

\begin{tabular}{|c|c|c|}
\hline Factor & Descripción & $\begin{array}{l}\text { Efecto sobre la tasa de } \\
\text { crecimiento del empleo }\end{array}$ \\
\hline Tamaño de la empresa & $\begin{array}{l}\text { De acuerdo con el número de } \\
\text { empleados }\end{array}$ & - \\
\hline Edad de la empresa & En años & - \\
\hline \multicolumn{3}{|l|}{ Estrategias de la empresa: } \\
\hline Innovación & $\begin{array}{l}\text { La introducción de nuevos } \\
\text { productos o procesos de } \\
\text { producción }\end{array}$ & + \\
\hline $\begin{array}{l}\text { Orientación de la empresa } \\
\text { hacia la exportación }\end{array}$ & $\begin{array}{l}\text { Realizar ventas de algunos } \\
\text { productos en los mercados } \\
\text { internacionales }\end{array}$ & + \\
\hline Intensidad del capital & $\begin{array}{c}\text { El nivel de capital y/o activos } \\
\text { fijos }\end{array}$ & + \\
\hline \multicolumn{3}{|c|}{ Otras características de la empresa } \\
\hline Localización & $\begin{array}{c}\text { Si la empresa está localizada } \\
\text { en un área densamente poblada } \\
\text { (p.e. una gran ciudad) }\end{array}$ & 0 \\
\hline Capital Humano & $\begin{array}{l}\text { Conocimientos y habilidades } \\
\text { adquiridos de los trabajadores a } \\
\text { través del entrenamiento formal }\end{array}$ & + \\
\hline Propiedad & $\begin{array}{c}\text { Si la empresa tiene propietarios } \\
\text { extranjeros }\end{array}$ & + \\
\hline $\begin{array}{l}\text { Orientación del sector hacia } \\
\text { la exportación }\end{array}$ & $\begin{array}{l}\text { La participación en la producción } \\
\text { de un sector que exporta }\end{array}$ & + \\
\hline
\end{tabular}




\begin{tabular}{|c|c|c|}
\hline Factor & Descripción & $\begin{array}{l}\text { Efecto sobre la tasa de } \\
\text { crecimiento del empleo }\end{array}$ \\
\hline \multicolumn{3}{|c|}{ Características propias: } \\
\hline Edad del propietario & $\begin{array}{c}\text { La edad del propietario de la } \\
\text { empresa en años }\end{array}$ & + \\
\hline Capital humano & $\begin{array}{l}\text { Conocimientos y habilidades } \\
\text { adquiridos por los trabajadores } \\
\text { a través de los estudios, el } \\
\text { entrenamiento y/o experiencia } \\
\text { previa en los negocios }\end{array}$ & Mixto \\
\hline
\end{tabular}

Fuente: De Kok, et al. (2013).

En la tabla 3, podemos ver que los factores que se encuentran relacionados positivamente con la tasa de crecimiento del empleo son: la innovación, la orientación hacia la exportación, capital humano, propiedad, sector exportador y la edad del propietario de la empresa en años.

Estudios recientes han encontrado una relación positiva entre el crecimiento del empleo y la innovación, la orientación a la exportación y la intensidad de capital. En cuanto a la edad del propietario los hallazgos muestran que las empresas dirigidas por empresarios de mayor edad crecen más rápido que las administradas por los propietarios más jóvenes (Kok, et al., 2013), lo anterior se debería a lo valioso que es la experiencia en el negocio.

\section{El medio ambiente de negocios y la generación de empleos}

Los factores del medio ambiente que más impacto tienen en el crecimiento del empleo de las PYME, lo vemos en la tabla siguiente:

TTabla 4.

\section{Factores que influyen en la generación de empleos de la PYME}

\begin{tabular}{|c|c|c|}
\hline Factor & Descripción & $\begin{array}{l}\text { Efecto sobre la tasa de } \\
\text { crecimiento del empleo }\end{array}$ \\
\hline Acceso a financiamiento & $\begin{array}{l}\text { La facilidad de las PYME para obtener un } \\
\text { préstamo }\end{array}$ & + \\
\hline Calidad de la infraestructura & $\begin{array}{l}\text { La confiabilidad de contar con una buena y } \\
\text { adecuada infraestructura física. }\end{array}$ & + \\
\hline $\begin{array}{l}\text { Regulaciones simples para } \\
\text { las empresas }\end{array}$ & $\begin{array}{l}\text { El tiempo promedio que una PYME necesita } \\
\text { para realizar trámites ante las entidades ofi- } \\
\text { ciales del gobierno y para adherirse a todas } \\
\text { las regulaciones. }\end{array}$ & + \\
\hline
\end{tabular}




\begin{tabular}{|c|l|c|}
\hline \multicolumn{1}{c|}{ Factor } & \multicolumn{1}{c|}{$\begin{array}{c}\text { Descripción } \\
\text { Bajo nivel de corrupción }\end{array}$} & $\begin{array}{l}\text { La frecto sobre la tasa de } \\
\text { a oficiales corruptos }\end{array}$ \\
\hline crecimiento del empleo
\end{tabular}

Fuente: De Kok, et al. (2013).

Como podemos ver en la tabla 4, las PYME que cuentan con acceso a financiamientos, una infraestructura confiable y adecuada que facilite su operación, facilidad para cumplir con las regulaciones gubernamentales y un bajo nivel de corrupción, podrán ser competitivas, generar y mantener los empleos.

\section{Evolución de la Política Empresarial en México}

Las políticas para las empresas en México se han transformado de manera gradual en una política de fomento empresarial que verdaderamente impulsen a este sector. En los últimos 20 años se ha puesto énfasis en la recuperación de la competitividad perdida por el proceso de apertura comercial, dado que la globalización económica actual ha cambiado sustancialmente el mercado natural de las PYMES mexicanas, pues la apertura comercial no ha servido como motor del crecimiento del grueso de las PYMES mexicanas (Balderas, et al., 2004).

A continuación se presenta en la tabla 5, una breve evolución de la política para las empresas:

TTabla 5.

Evolución de la política empresarial en México

\begin{tabular}{|l|l|l|l|}
\hline \multicolumn{1}{|c|}{ Periodo } & \multicolumn{1}{c|}{ Objetivos } & \multicolumn{1}{c}{ Acciones } \\
\hline & & $\begin{array}{l}\text { Eliminar los obstáculos } \\
\text { que impedian el funcio- } \\
\text { namiento del mercado, } \\
\text { creando las condiciones }\end{array}$ \\
\hline $\begin{array}{l}\text { 1989-1994: la aper- } \\
\text { tura económica y el } \\
\text { período de transición }\end{array}$ & $\begin{array}{l}\text { Programa nacional de } \\
\text { ciencia y modernización } \\
\text { tecnológica 1990-1994. }\end{array}$ & $\begin{array}{l}\text { saran a las unida-des } \\
\text { productivas a integrar-se } \\
\text { a los procesos mundiales y } \\
\text { hacer del cambio técnico } \\
\text { un elemento central de su } \\
\text { estrategia para competir } \\
\text { con ventaja. }\end{array}$ \\
\hline
\end{tabular}




\begin{tabular}{|c|c|c|c|}
\hline Período & Programas & Objetivos & Acciones \\
\hline $\begin{array}{l}\text { 1995-2000: el nuevo } \\
\text { marco normativo y } \\
\text { el tratado de libre } \\
\text { comercio de América } \\
\text { del Norte }\end{array}$ & $\begin{array}{l}\text { Programa Nacional de } \\
\text { Industria y Comercio } \\
\text { Exterior 1995-2000 } \\
\text { (PROPICE). } \\
\text { Prog r a m a de } \\
\text { Capacitación } \\
\text { Industrial de Mano de } \\
\text { Obra - CIMO. } \\
\text { Programa de Moderni- } \\
\text { zación Tecnológica del } \\
\text { Consejo Nacional de } \\
\text { Ciencia y Tecnología } \\
\text { (CONACYT). } \\
\text { Co mité Na cion nal } \\
\text { de Productividad e } \\
\text { Innovación Tecnológica } \\
\text { COMPITE. } \\
\text { Sistema de Información } \\
\text { Empresarial Mexicano } \\
\text { (SIEM). }\end{array}$ & $\begin{array}{l}\text { Promover la competitivi- } \\
\text { dad de la base industrial } \\
\text { en México, con especial } \\
\text { énfasis en las PYMES. }\end{array}$ & $\begin{array}{l}\text { Incrementar las ex- } \\
\text { portaciones directas e } \\
\text { indirectas con utilidades } \\
\text { altas y sostenibles. } \\
\text { Promover el desarrollo } \\
\text { de los mercados internos } \\
\text { y sustituir eficientemen- } \\
\text { te las importaciones. } \\
\text { Acelerar el desarrollo } \\
\text { industrial y regional al } \\
\text { igual que los agrupa- } \\
\text { mientos industriales. }\end{array}$ \\
\hline $\begin{array}{l}\text { 2001-2007: ¿hacia la } \\
\text { construcción de una } \\
\text { política integral? }\end{array}$ & $\begin{array}{l}\text { Ley para el desarrollo de } \\
\text { la competitividad de la } \\
\text { micro, pequeña y media- } \\
\text { na empresa (2002). }\end{array}$ & $\begin{array}{l}\text { Articular el conjunto de } \\
\text { políticas, estrategias, ac- } \\
\text { ciones e instrumentos } \\
\text { para apoyar la competiti- } \\
\text { vidad de las PYME. } \\
\text { Establecer las bases para } \\
\text { la planeación (federal y } \\
\text { estatal), formulación y } \\
\text { ejecución de las políticas } \\
\text { públicas. }\end{array}$ & $\begin{array}{l}\text { Creación de la } \\
\text { Subsecretaría para la } \\
\text { Pequeña y Mediana } \\
\text { Empresa (SPYME). } \\
\text { Puesta en marcha del } \\
\text { Fondo PYME. } \\
\text { Creación del Consejo } \\
\text { Nacional de la Micro, } \\
\text { Pequeña y Mediana } \\
\text { Empresa. }\end{array}$ \\
\hline
\end{tabular}




\begin{tabular}{|c|c|c|c|}
\hline Periodo & Programas & Objetivos & Acciones \\
\hline 2007-2012 & $\begin{array}{l}\text { Plan Nacional de desa- } \\
\text { rrollo 2007-2012 } \\
\text { Programa sectorial de } \\
\text { economía 2007-2012 }\end{array}$ & $\begin{array}{l}\text { Promover la creación, } \\
\text { desarrollo y consolidación } \\
\text { de las micro, pequeñas y } \\
\text { medianas empresas. } \\
\text { Contribuir a la generación } \\
\text { de empleos a través del } \\
\text { impulso a la creación de } \\
\text { nuevas empresas y la } \\
\text { consolidación de las } \\
\text { PYMES existentes. } \\
\text { Impulsar la generación } \\
\text { de más y mejores ocupa- } \\
\text { ciones entre la población } \\
\text { emprendedora de bajos } \\
\text { ingresos, mediante la pro- } \\
\text { moción y fortalecimiento } \\
\text { de proyectos productivos. }\end{array}$ & $\begin{array}{l}\text { Favorecer el incremento } \\
\text { de la productividad. } \\
\text { Consolidar lo esquemas } \\
\text { de apoyo a la PYME en } \\
\text { una sola instancia. } \\
\text { Impulsar el desarrollo de } \\
\text { proveedores. }\end{array}$ \\
\hline $\begin{array}{l}2013-2018 \\
\text { Actual }\end{array}$ & $\begin{array}{l}\text { PND 2013-2018 } \\
\text { Reglas de operación del } \\
\text { Fondo PYME }\end{array}$ & $\begin{array}{l}\text { Se busca el avance hacia } \\
\text { un modelo de crecimiento } \\
\text { cimentado en la inno- } \\
\text { vación, aprovechando } \\
\text { el impulso de las expor- } \\
\text { taciones y propiciando } \\
\text { mecanismos para aumen- } \\
\text { tar el contenido nacional } \\
\text { de las mismas }\end{array}$ & $\begin{array}{l}\text { Institut o de l } \\
\text { emprendedor } \\
\text { Fomento a la innovación } \\
\text { I mpulso a las } \\
\text { exportaciones } \\
\text { Consolidar encadena- } \\
\text { mientos productivos }\end{array}$ \\
\hline
\end{tabular}

Fuente: Elaboración propia con base en Brown, F. y Dominguez, L. (2010), Plan Nacional de Desarrollo 2007-2012 (DOF, 2007) y Programa sectorial de economía 2007-2012 (DOF, 2008).

En la tabla 5, podemos ver cómo la política ha evolucionado desde el fomento empresarial hasta el intento de contar con una política integral. De este modo, se cambia el enfoque de subsidios hacia la necesidad de apoyar a las cadenas productivas, a través de las cuales se busca la realización de transferencias de tecnologías mediante el establecimiento de vínculos entre clientes y proveedores. Poniendo así en primer plano la urgencia de atender el rezago de las empresas de menor tamaño. 
En el sexenio 2007-2012, la política se encontraba diseñada con base en el Plan Nacional de Desarrollo 2007-2012 y el Programa Sectorial de Economía 2007-2012. Para operar esta política se creó la Subsecretaria para la Pequeña y Mediana Empresa (SPYME). Ambos programas el Nacional y el Sectorial, proponían promover la creación, desarrollo y consolidación de las PYME. Mostraban una política más definida que las anteriores con respecto a las PYME, sin embargo, no estipulan una política empresarial sectorial, es decir, no distinguía entre los sectores: industria, comercio, servicios, agrícola, artesanal, etc., que cuentan con necesidades diferenciadas y deberían contar con estrategias específicas para su fomento. La estrategia de atención de estos programas proponían beneficiarios claramente focalizados por su tamaño y potencial: en emprendedores, microempresas, pequeñas y medianas, empresas gacela ${ }^{1}$ y empresas tractoras ${ }^{2}$.

Esta política era operada con dos servicios principales, por un lado el acceso al financiamiento y por otro lado el desarrollo empresarial con servicios de capacitación, comercialización, gestión e innovación en centros de asistencia integral como México Emprende 3 , los cuales intentaban resolver el problema del escaso financiamiento involucrando a la banca de desarrollo y la banca comercial, con una novedosa estrategia de acercamiento entre la banca comercial y el empresario a través de la intervención de un consultor financiero encargado de guiar al empresario durante el trámite del crédito. Y con el impulso al Programa de garantías, con el fin de subsanar la principal limitación del empresario al buscar un financiamiento: la falta de garantías.

\footnotetext{
1. "Gacela" son aquellas empresas que tienen que cumplir con al menos 3 años de operaciones y generar un crecimiento superior al 15\% en ventas en los 3 últimos años. La mayoría de éstas son empresas innovadoras y de base tecnológica que tienen altas probabilidades de crecer a nivel nacional e internacional. Generalmente se trata de empresas de tamaño mediano y grande.

2. Las empresas tractoras son todas aquellas grandes empresas que fomentan el crecimiento y desarrollo económico a nivel Macro, ya que la mayoría de ellas son grandes firmas a nivel nacional o internacional. Las empresas tractoras de México representan un mercado fundamental para el crecimiento y apuntalamiento de las pequeñas y medianas empresas (PYME), ya que existen programas como por ejemplo el Desarrollo de proveedores, a través de las cuales las empresas tractoras impulsan a las micro y pequeñas convirtiéndolas en las proveedoras de los insumos que utiliza.

3. Son oficinas autorizadas por el Gobierno Federal en México y creadas para el otorgamiento de servicios y apoyos públicos o privados para las PYME, de manera integral, accesible, ágil y oportuna, de acuerdo con su tamaño y potencial.
}

\section{Política PYME actual (2013-2018)}

Con el objetivo de seguir impulsando la creación de más y mejores Micro, Pequeñas y Medianas Empresas, la Secretaría de Economía publicó el 15 de abril de 2013, en el Diario Oficial de la Federación el decreto mediante el cual se crea el Instituto Nacional del Emprendedor (INADEM) (DOF, 2013).

Este nuevo Instituto busca que México avance hacia un modelo de crecimiento cimentado en la innovación, aprovechando el impulso de las exportaciones y propiciando mecanismos para aumentar el contenido nacional de las mismas. Además busca beneficiar al mercado interno, favoreciendo la consolidación de los encadenamientos productivos.

Las líneas estratégicas que seguirá el INADEM serán:

- Impulsar sectores estratégicos con alto potencial de crecimiento, generación de empleo, y participación de valor agregado en las cadenas de exportación;

- Favorecer el desarrollo regional potenciando las vocaciones productivas y las ventajas competitivas, brindando especial atención a aquellas con problemáticas sociales y económicas más sentidas y claramente detectadas;

- Fortalecer el Ecosistema de Financiamiento de acuerdo con las prioridades regionales y sectoriales, garantizando que llegue efectivamente a los emprendedores y a las PYME.

- Propiciar el crecimiento basado en la innovación que genere mayor riqueza y empleo bien remunerado, así como incidir en la reconstrucción y fortalecimiento del tejido social.

Este nuevo Instituto Nacional del Emprendedor es la instancia pública de vanguardia, especializada, cercana al interés de los emprendedores, las micro, pequeñas y medianas empresas y está encargado de trabajar en la construcción de un escenario fértil, alejado de regulaciones excesivas y obstáculos burocráticos.

En México el CONEVAL es el que se encarga de medir el impacto de las políticas en el desarrollo de las PYME, sin embargo, en las diversas evaluaciones que ha realizado no ha podido determinar este impacto 
dado que los indicadores no son contundentes y no se cuenta con una metodología adecuada de evaluación (Saavedra, Tapia y Anaya, 2013).

\section{Metodología}

Este trabajo es de tipo descriptivo, los datos fueron recolectados a través de investigación documental de fuentes primarias y secundarias de información. Se ha realizado un análisis longitudinal con el fin de establecer la evolución de la PYME utilizando dos indicadores: Número de establecimientos y personal ocupado, con un estudio general y sectorial. Los años de estudio fueron tomados a partir de los censos económicos de INEGI, por esa razón el análisis solo abarca hasta el año 2008, que fue el último censo económico en México.

\section{Análisis y evolución del sector empresarial en México}

Con el fin de comprender cuál ha sido la evolución del sector empresarial, específicamente un sector tan importante en México como la PYME, a continuación presentamos un conjunto de tablas con un breve análisis de la tendencia que ha seguido este sector empresarial.

Podemos ver en la tabla 6 la tendencia del número de establecimientos de acuerdo con el tamaño de empresa desde 1994 hasta el último censo económico que se realizó en el año 2009 donde los datos que se recolectaron corresponden al año 2008.

ITabla 6.

\section{Número de establecimientos por tamaño de empresa}

\begin{tabular}{|l|c|c|c|c|c|c|c|c|c|}
\hline Sector & 1994 & $\%$ & 1998 & $\%$ & 2003 & $\%$ & 2008 & $\%$ & $\begin{array}{c}\text { Crec. } \\
\text { Prom. }\end{array}$ \\
\hline $\begin{array}{l}\text { Pro9-2008 } \\
\text { anual }\end{array}$ \\
\hline Total & $2,150.20$ & 100.00 & $2,726.40$ & 100.0 & $2,912.60$ & 100.0 & $3,656.00$ & 100.0 & $4.8 \%$ \\
\hline Micro & $2,139.90$ & 97.70 & $2,614.90$ & 95.9 & $2,783.50$ & 95.6 & $3,496.70$ & 95.6 & $4.5 \%$ \\
\hline Pequeña & 35.90 & 1.60 & 83.90 & 3.1 & 95.70 & 3.3 & 122.50 & 3.4 & $17.2 \%$ \\
\hline Mediana & 6.20 & 0.30 & 19.20 & 0.7 & 22.30 & 0.8 & 24.60 & 0.7 & $21.2 \%$ \\
\hline PYME & $2,182.00$ & 99.80 & $2,718.00$ & 99.7 & $2,901.50$ & 99.70 & $3,643.80$ & 99.70 & $4.8 \%$ \\
\hline Grande & 3.30 & 0.20 & 8.40 & 0.3 & 11.20 & 0.30 & 12.20 & 0.30 & $19.3 \%$ \\
\hline
\end{tabular}

Fuente: Elaboración propia con base en Censos Económicos INEGI (1994, 1998, 2003 2010). 
Observamos que la microempresa tuvo un crecimiento promedio anual de $4.5 \%$ la pequeña empresa reportó un crecimiento de $17.2 \%$ y la mediana empresa tuvo un crecimiento aún más elevado de $21.2 \%$; de este modo las PYME en promedio crecieron sólo un $4.8 \%$ manteniendo una participación relativamente estable en $99.70 \%$ del total de las empresas, mientras que la empresa grande creció a una tasa más elevada alcanzando un $19.3 \%$ anual, aumentado su participación de $0.20 \%$ a $0.30 \%$ del total de las empresas incremento que represento cerca de 9 mil empresas más. Así pues, la tendencia de crecimiento no es favorable para la microempresa que tan sólo creció un $4.5 \%$ promedio anual.

Con respecto a la generación de empleos podemos ver en la tabla 7 que dentro del sector de PYME la microempresa es la que reporta una tasa más alta de crecimiento en este indicador $5.6 \%$, refrendando con esto su aporte a la generación de empleos al ubicarse en sectores económicos intensivos en mano de obra, permitiendo así una mejor distribución del ingreso.

-Tabla 7.

Personal ocupado por tamaño de empresas

\begin{tabular}{|l|c|c|c|c|c|c|c|c|c|}
\hline Sector & 1994 & $\%$ & 1998 & $\%$ & 2003 & $\%$ & 2008 & $\%$ & $\begin{array}{c}\text { Crec. Prom. } \\
\text { 1994-2008 } \\
\text { anual }\end{array}$ \\
\hline Total & $9,385.50$ & 100.0 & $11,937.80$ & 100.0 & $14,136.0$ & 100.0 & $17,663.6$ & 100.0 & $6.3 \%$ \\
\hline Micro & $4,633.40$ & 49.4 & $5,180.30$ & 42.1 & $6,032.1$ & 42.7 & $8,236.1$ & 46.6 & $5.6 \%$ \\
\hline Pequeña & $1,349.90$ & 14.4 & $1,657.10$ & 13.1 & $1,870.2$ & 13.2 & $2,172.4$ & 12.3 & $4.4 \%$ \\
\hline Mediana & $1,570.20$ & 16.7 & $1,545.90$ & 11.7 & $1,679.5$ & 11.9 & $1,808.3$ & 10.2 & $1.1 \%$ \\
\hline PYME & $7,553.50$ & 80.50 & $8,383.30$ & 66.9 & $9,581.80$ & 67.8 & $12,216.8$ & 69.1 & $4.4 \%$ \\
\hline Grande & $1,831.80$ & 19.5 & $3,554.40$ & 33.1 & $4,554.20$ & 32.2 & $5,446.8$ & 30.8 & $14.1 \%$ \\
\hline
\end{tabular}

Fuente: Elaboración propia con base en Censos Económicos INEGI (1994, 1998, 2003 2010).

Sin embargo, se puede observar también en la tabla 7 que el sector PYME ha ido perdiendo participación como empleadora con respecto a la empresa grande y ha disminuido de $80.50 \%$ en 1994 a $69.19 \%$ en 2008. Esta disminución se debe al bajo nivel de la tasa de crecimiento promedio anual, que para las PYME es de tan sólo 4.4\%, mientras que la empresa grande reporta un crecimiento de $14.1 \%$, y su aportación a la generación de empleo cambio de $19.5 \%$ a $30.8 \%$.
En cuanto al comportamiento sectorial de los indicadores más importantes: número de establecimientos y personal ocupado, empezamos analizando el sector manufacturero, uno de los más importantes dados que a pesar de que las PYME manufactureras concentraron solo el $11.7 \%$ de unidades económicas de acuerdo con el Censo Económico de INEGI (2010), es el que más valor agregado genera. Dado el alto consumo de mano de obra y materias primas por lo general de origen nacional. 
Tabla 8.

Manufacturas: Personal ocupado por tamaño (Miles de personas y porcentajes)

\begin{tabular}{|l|c|c|c|c|c|c|c|c|c|}
\hline Sector & 1994 & $\%$ & 1998 & $\%$ & 2003 & $\%$ & 2008 & $\%$ & $\begin{array}{c}\text { Crec. Prom. } \\
1994-2008 \\
\text { anual }\end{array}$ \\
\hline Total & $3,263.80$ & 100.0 & $4,232.30$ & 100.0 & $4,198.60$ & 100.0 & $4,552.80$ & 100.0 & $2.8 \%$ \\
\hline Micro & 815.00 & 25.0 & 773.30 & 18.3 & 762.10 & 18.1 & $1,075.90$ & 23.8 & $2.3 \%$ \\
\hline Pequeña & 583.40 & 17.9 & 499.50 & 11.8 & 431.80 & 10.3 & 460.20 & 6.7 & $-1.5 \%$ \\
\hline Mediana & 939.50 & 28.8 & 916.70 & 21.7 & 810.10 & 19.3 & 765.90 & 9.1 & $-1.3 \%$ \\
\hline PYME & $2,337.90$ & 71.7 & $2,189.50$ & 51.80 & $2,004.00$ & 47.7 & $2,302.00$ & 36.6 & $-0.1 \%$ \\
\hline Grande & 925.80 & 28.3 & $2,042.90$ & 48.20 & $2,194.60$ & 52.3 & $2,220.80$ & 60.4 & $10.0 \%$ \\
\hline
\end{tabular}

Fuente: Elaboración propia con base en Censos Económicos INEGI (1994, 1998, 2003 2010).

Como podemos ver en la tabla 8, las PYME manufactureras sufren un decrecimiento alarmante en su participación con relación a la generación de empleos pues de tener $71.70 \%$ en 1994 cambia a $39.60 \%$ en 2008 , lo que ocasiona que la empresa grande de este sector, sea más importante en este indicador, pues reportó un crecimiento considerable al pasar de $28.4 \%$ a $60.4 \%$, con un crecimiento promedio anual de $10 \%$.

Así también, vemos que la micro empresa se ha mantenido puesto que su participación disminuyó solo 1.2 puntos porcentuales, mientras que la pequeña empresa lo hizo en 11.2 puntos porcentuales y la mediana empresa en 19.7 puntos porcentuales. Es importante señalar que la productividad de las PYME en el sector industrial es baja debido a que utilizan procesos manuales y artesanales en su proceso productivo, mientras que la empresa grande está altamente tecnificada, esto le permite producir con economías de escala.
El crecimiento que ha tenido la empresa industrial grande, en la generación de empleos se puede explicar por las propias características del sector industrial de ser intensivo en tecnología y capital, aspectos que difícilmente pueden mantener Las empresas PYME. Lo anterior sucede debido a que este es un sector dual; por un lado, empresas grandes, normalmente transnacionales con tecnologías modernas, productoras de bienes durables; $y$, por el otro, un sector de empresas más pequeñas principalmente nacionales con escasa tecnología y baja productividad, dedicadas a la producción de bienes no durables, destinados al mercado interno (Hualde,1998).

Con respecto al número de establecimientos podemos ver en la tabla 9 como la participación de las PYME manufactureras se han mantenido estable con un crecimiento promedio de $4.6 \%$ anual, superior al crecimiento de la empresa grande que sólo creció a una tasa de $2.2 \%$ anual. 
-Tabla 9.

Manufacturas: Número de establecimientos por tamaño (Miles de establecimientos y porcentajes)

\begin{tabular}{|c|c|c|c|c|c|c|c|c|c|}
\hline Sector & 1994 & $\%$ & 1998 & $\%$ & 2003 & $\%$ & 2008 & $\%$ & $\begin{array}{l}\text { Crec. Prom. } \\
\text { 1994-2008 } \\
\text { anual }\end{array}$ \\
\hline Total & 266.00 & 100.00 & 344.10 & 100.0 & 328.70 & 100.0 & 435.4 & 100.0 & $4.5 \%$ \\
\hline Micro & 248.10 & 93.3 & 310.10 & 90.1 & 298.70 & 90.9 & 403.5 & 92.7 & $4.5 \%$ \\
\hline Pequeña & 12.50 & 4.7 & 22.70 & 6.6 & 19.80 & 6.0 & 22.1 & 5.0 & $5.5 \%$ \\
\hline Mediana & 3.10 & 1.2 & 8.20 & 2.4 & 7.20 & 2.2 & 6.8 & 1.6 & $8.5 \%$ \\
\hline PYME & 263.70 & 99.20 & 341.00 & 99.1 & 325.70 & 99.10 & 432.40 & 99.30 & $4.6 \%$ \\
\hline Grande & 2.30 & 0.8 & 3.0 & 0.9 & 3.10 & 0.9 & 3.0 & 0.7 & $2.2 \%$ \\
\hline
\end{tabular}

Fuente: Elaboración propia con base en Censos Económicos INEGI (1994, 1998, 2003, 2010).

El hecho de que el número de las PYME industriales se haya mantenido estable y que a pesar de esto no haya sido capaz de mantener los empleos es preocupante, pues implica que se están creando empresas industriales con baja inversión fija, lo que genera baja productividad y capacidad de innovación y creación de empleos, aspectos fundamentales para mantenerse en un mercado de libre competencia como el mexicano (Pavón, 2010).

En cuanto al sector comercio de acuerdo con los Censos Económicos de INEGI (2010), el 50\% de las unidades económicas pertenecen a este sector, las cuales como podemos observar en la tabla 10 generaron empleo a una tasa promedio anual de 5.5\%, siendo en este segmento la empresa comercial de tamaño micro la que reportó mayor crecimiento con un $7.5 \%$ promedio anual, mientras que la empresa pequeña quedó en desventaja al decrecer un $0.5 \%$. Si comparamos con la empresa grande, en este sector el crecimiento fue muy superior al alcanzar un $14.6 \%$ promedio anual. Es importante señalar "que en este sector las PYME pagan mejores remuneraciones a sus empleados dada su elevada productividad originada por una inversión en activos fijos superior a la de otros sectores productivos" (Pavón, 2010, p. 20).

Con respecto al número de establecimientos en el sector comercio, observamos en la tabla 11 que el $99.90 \%$ son PYME al año 2008, y han mantenido su participación con respecto a la empresa grande; sin embargo, en cuanto al crecimiento en unidades económicas dichas empresas reportan un promedio anual de $3.9 \%$, siendo en este segmento que las que reportan mayor crecimiento son las empresas de tamaño mediano. Por lo que respecta a la empresa grande del sector comercial, esta reporta un crecimiento muy elevado el cual alcanza en promedio $67.9 \%$ anual para el período bajo estudio, lo que implica un mayor dinamismo para enfrentar los embates de las crisis económicas en comparación con las empresas de menor tamaño. 
TTabla 10.

Comercio: Personal ocupado por tamaño de empresa (Miles de personas y porcentajes)

\begin{tabular}{|l|c|c|c|c|c|c|c|c|c|}
\hline Sector & 1994 & $\%$ & 1998 & $\%$ & 2003 & $\%$ & 2008 & $\%$ & $\begin{array}{c}\text { Crec. Prom. } \\
\text { 1994-2008 } \\
\text { anual }\end{array}$ \\
\hline Total & $3,217.70$ & 100.0 & $3,784.90$ & 100.0 & $4,997.4$ & 100.0 & $6,183.60$ & 100.0 & $6.6 \%$ \\
\hline Micro & $1,950.70$ & 60.6 & $2,490.50$ & 65.8 & $3,100.9$ & 62.1 & $4,004.90$ & 64.8 & $7.5 \%$ \\
\hline Pequeña & 470.40 & 14.6 & 433.30 & 11.4 & 557.6 & 11.2 & 436.90 & 7.1 & $-0.5 \%$ \\
\hline Mediana & 413.80 & 12.9 & 369.40 & 9.8 & 512.3 & 10.3 & 577.80 & 9.3 & $2.8 \%$ \\
\hline PYME & $2,834.90$ & 88.1 & $3,293.20$ & 87.0 & $4,170.8$ & 83.6 & $5,019.60$ & 81.2 & $5.5 \%$ \\
\hline Grande & 382.80 & 11.9 & 491.60 & 13.0 & 826.6 & 16.4 & $1,164.00$ & 18.8 & $14.6 \%$ \\
\hline
\end{tabular}

Fuente: Elaboración propia con base en Censos Económicos INEGI (1994, 1998, 2003 2010).

Tabla 11.

Comercio: Número de establecimientos por tamaño de empresa (Miles de establecimientos y porcentajes)

\begin{tabular}{|l|c|c|c|c|c|c|c|c|c|}
\hline Sector & 1994 & $\%$ & 1998 & $\%$ & 2003 & $\%$ & 2008 & $\%$ & $\begin{array}{c}\text { Crec. Prom. } \\
\text { 1994-2008 } \\
\text { anual }\end{array}$ \\
\hline Total & $1,210.20$ & 100.00 & $1,443.70$ & 100.0 & $1,580.60$ & 100.0 & $1,869.10$ & 100.0 & $3.9 \%$ \\
\hline Micro & $1,197.20$ & 98.9 & $1,408.60$ & 97.6 & $1,533.90$ & 97.0 & $1,816.40$ & 97.2 & $3.7 \%$ \\
\hline Pequeña & 11.20 & 0.90 & 25.50 & 1.8 & 33.00 & 2.1 & 37.40 & 2.0 & $16.7 \%$ \\
\hline Mediana & 1.50 & 0.10 & 7.30 & 0.5 & 10.00 & 0.6 & 11.10 & 0.6 & $45.7 \%$ \\
\hline PYME & $1,209.90$ & 99.90 & $1,441.40$ & 99.9 & $1,576.90$ & 99.70 & $1,864.90$ & 99.8 & $3.9 \%$ \\
\hline Grande & 0.40 & 0.10 & 2.30 & 0.1 & 3.70 & 0.30 & 4.20 & 0.2 & $67.9 \%$ \\
\hline
\end{tabular}

Fuente: Elaboración propia con base Censos Económicos INEGI (1994, 1998, 2003, 2010). 
De acuerdo con el Censo Económico INEGI (2010), las unidades económicas del sector servicios representaron el $36.2 \%$ del total nacional y el personal ocupado representó el $34.4 \%$ situándose en primer lugar como empleador. Como podemos ver en la tabla 12, ha sido la pequeña empresa la que mayor crecimiento en el empleo ha reportado con una tasa promedio anual de $23.6 \%$, aún más elevado que el crecimiento reportado por la empresa grande que alcanzó el $21 \%$ anual en el período bajo estudio. Este sector son importantes los negocios de hoteles y restaurantes, pues ocupan el primer lugar en la generación de empleos, esto se explica cuando vemos que el sector turístico es uno de los sectores económicos más importantes en México, comprobándose esto en la generación de ingresos, empleos y divisas.

TTabla 12.

Servicios: Personal ocupado por tamaño de empresa (Miles de personas y porcentajes)

\begin{tabular}{|l|c|c|c|c|c|c|c|c|c|}
\hline Sector & 1994 & $\%$ & 1998 & $\%$ & 2003 & $\%$ & 2008 & $\%$ & $\begin{array}{c}\text { Crec. Prom. } \\
\text { 1994-2008 } \\
\text { anual }\end{array}$ \\
\hline Total & $2,904.00$ & 100.00 & $3,920.60$ & 100.0 & $4,940.00$ & 100.0 & $6,957.20$ & 100.0 & $10.0 \%$ \\
\hline Micro & $1,867.70$ & 64.3 & $1,916.50$ & 48.9 & $2,169.10$ & 43.9 & $3,155.30$ & 45.3 & $4.9 \%$ \\
\hline Pequeña & 296.10 & 10.2 & 724.30 & 18.5 & 880.80 & 17.9 & $1,275.30$ & 18.3 & $23.6 \%$ \\
\hline Mediana & 216.90 & 7.5 & 259.80 & 6.6 & 357.10 & 7.2 & 464.60 & 6.7 & $8.2 \%$ \\
\hline PYME & $2,380.70$ & 82.0 & $2,900.60$ & 74.0 & $3,407.00$ & 69.0 & $4,895.20$ & 70.3 & $7.5 \%$ \\
\hline Grande & 523.20 & 18.0 & $1,019.90$ & 26.0 & $1,533.00$ & 31.0 & $2,062.00$ & 29.7 & $21.0 \%$ \\
\hline
\end{tabular}

Fuente: Elaboración propia con base en Censos Económicos INEGI (1994, 1998, 2003, 2010).

Sin embargo, podemos observar en la tabla 12 que, a pesar del alto crecimiento en el personal ocupado sobre todo de la empresa pequeña, su contribución al empleo total ha disminuido; en el año 1994 representaba un $82 \%$ del total, mientras que en 2008 reporta una disminución con lo que se coloca en $70.30 \%$; la empresa grande es la que gana participación, pues en este mismo período pasa de $18 \%$ a casi $30 \%$ de la participación total en la generación de empleo de este sector.
En cuanto al número de establecimientos; la participación de las PYME del sector servicios se ha mantenido, como podemos observar en la tabla 13, sin embargo, la tasa de crecimiento promedio anual para este período es $6.4 \%$, mientras que la empresa grande creció a un ritmo de $52 \%$ anual, mostrando con esto un dinamismo más alto y una fuerte capacidad para enfrentarse al entorno adverso por el que ha pasado el país en los últimos años. 
Tabla 13.

\section{Servicios: Número de establecimientos por tamaño de empresa (Miles de establecimientos y porcentajes)}

\begin{tabular}{|c|c|c|c|c|c|c|c|c|c|}
\hline Sector & 1994 & $\%$ & 1998 & $\%$ & 2003 & $\%$ & 2008 & $\%$ & $\begin{array}{l}\text { Crec. Prom. } \\
1994-2008\end{array}$ \\
\hline Total & 709.00 & 100.0 & 938.60 & 100.0 & $1,003.30$ & 100.0 & $1,351.50$ & 100.0 & $6.5 \%$ \\
\hline Micro & 694.60 & 98.0 & 896.20 & 95.5 & 950.90 & 94.8 & $1,276.80$ & 94.5 & $6.0 \%$ \\
\hline Pequeña & 12.20 & 1.7 & 35.60 & 3.8 & 42.90 & 4.3 & 63.00 & 4.6 & $29.7 \%$ \\
\hline Mediana & 1.60 & 0.20 & 3.70 & 0.4 & 5.10 & 0.5 & 6.70 & 0.5 & $22.8 \%$ \\
\hline PYME & 708.40 & 99.90 & 935.50 & 99.7 & 998.90 & 99.6 & $1,346.50$ & 99.6 & $6.4 \%$ \\
\hline Grande & 0.60 & 0.1 & 3.10 & 0.3 & 4.40 & 0.4 & 5.00 & 0.4 & $52.4 \%$ \\
\hline
\end{tabular}

Fuente: Elaboración propia con base en Censos Económicos INEGI (1994, 1998, 2003 2010).

Cómo hemos podido observar en las tablas del 4 al 13, la tendencia respecto del número de unidades económicas y generación de empleos de las PYME de los tres sectores es a la baja, ganando un importante terreno la empresa grande, lo que estaría indicando que las últimas reformas económicas y políticas implementadas no se han realizado de acuerdo con las necesidades de las PYME mexicana. Más alarmante aún es ver cómo el sector más afectado es el industrial, pues las políticas industriales se han generado e implementado para las empresas grandes, que por lo general son exportadoras, en detrimento del sector industrial micro que tiene necesidades diferentes y ha sido olvidado.

\section{Resultados}

Como resultado de haber realizado un análisis de la evolución del sector empresarial mexicano, se tiene que :

- En cuanto al número de establecimientos por tamaño de empresas destacan el crecimiento de la empresa mediana y grande con tasas anuales de alrededor de $20 \%$, mientras que empresa micro solo logra obtener un $4 \%$ de crecimiento anual, mostrando con esto las grandes dificultades que encuentra esta última para sobrevivir.

- En el aspecto de generación de empleos a pesar que la mayor proporción lo muestran las empresas de tamaño micro esta ha ido perdiendo participación al pasar de $49 \%$ a $46 \%$ en el período analizado en beneficio de la empresa grande que ha ido aumentando su participación al pasar de $19 \%$ a $30 \%$, debiéndose esto a que las empresas de menor tamaño han sido vulnerables ante un entorno incierto y no han sido capaces de aumentar sino que todo lo contrario tienden a disminuir. - En el sector industrial la pérdida de participación en la generación de empleos es drástica sobre todo para la mediana empresa que pasa de una participación de $28 \%$ a tan solo $9 \%$, mientras que la empresa grande ha duplicado su participación al pasar de $28 \%$ a $60 \%$, debiéndose esto a que las políticas implementadas para apoyar al sector no han sido capaces de impulsar a las PYME para alcanzar un nivel de productividad para competir en el mercado, siendo notorio esto en el análisis que se hace respecto del número de unidades económicas, misma que no ha sufrido grandes variaciones. 
- El sector comercio también muestra pérdida de participación respecto de la generación de empleos siendo más notorio en la empresa pequeña, ganando terreno la empresa grande, siendo muy pocos los cambios que se observan en cuanto al número de establecimientos, mostrando con esto una vez más al alta competitividad de la empresa grande.

- El sector servicios se mostró como uno de los sectores más dinámicos en cuanto a la generación de empleos sobre todo en las empresas de tamaño pequeño, sin embargo también ha ido perdiendo participación y cediéndole el terreno a la empresa grande, siendo que los cambios en el número de establecimientos no se muestran tan drásticos.

\section{Conclusiones}

Es así como el análisis de esta problemática y evolución de las PYME, nos permite ver que las PYME son una fuente importante de generación de empleos en México, sin embargo su participación ha ido en declive en el periodo analizado (1994-2008) siendo el sector industrial es el que más terreno ha perdido; esto nos permite comprender que no es suficiente implementar políticas en el nivel macro a las que por lo general sólo tienen alcance y compresión las empresas medianas y pequeñas, siendo que una gran proporción son empresas de tamaño micro, las cuales no cuentan con la suficiente estructura para acceder a los apoyos implementados por el Gobierno Federal. $\mathrm{Si}$ bien es cierto no ha sido posible establecer una relación entre los indicadores de crecimiento de las PYME y los indicadores que sirven para evaluar las Política empresariales, en razón de que estos últimos no resultan claros no consistentes, basta con ver el detrimento en la evolución de las PYME tanto a nivel de número de unidades como de generación de empleos para darnos cuenta que las políticas implementadas no han funcionado para fomentar a las PYME en México.

Se podría de algún modo tomar en consideración este breve diagnóstico con el fin de sugerir algunos aspectos que se deben tomar en cuenta para modificar el rumbo de las políticas y de este modo alcanzar a las empresas que realmente necesitan el apoyo, o sea las microempresas:
- Se deben implementar políticas diferenciando los sectores (industria, comercio y servicios) dado que los mismos son diferentes y se deben especificar los apoyos de financiamiento y capacitación considerando este aspecto.

- Fortalecer la vinculación con el sector educativo a fin de generar tecnología propia acorde a sus características y necesidades, así como propiciar la innovación necesaria en todos los sectores de las PYME.

- Mejorar las regulaciones sobre todo en materia de competencia dado que existen monopolios que no se han logrado erradicar y que compiten en ventaja frente a las PYME mexicanas.

- Capacitar a verdaderos consultores de las PYME, pues la mayoría intentan implementar en las empresas modelos que han sido creados para las empresas grandes de otros contextos culturales y económicos, mismo que al no ser adaptados antes corren el riesgo de fracasar.

- Fomentar la cultura empresarial sobre todo en el empresario del segmento de las microempresas, pues la profesionalización de su administración es un aspecto que le genera muchas limitantes.

- Comunicar por los canales adecuados la existencia de los programas de apoyo que se han implementado para este sector empresarial, evitando así que sólo algunos privilegiados se beneficien, por un lado; y por el otro, evitando que sean timados por instituciones fraudulentas, que de modo ilegal obtienen los apoyos y los canalizan a los empresarios cobrándoles tasas más elevadas que las que les corresponde pagar.

En este sentido Dussel (2004) ha señalado que "el fomento a las empresas de menor tamaño requieren de una visión sistémica de competitividad mediante instrumentos regionales-sectoriales de largo plazo y en forma conjunta entre los sectores público y privado (p.72). Atendiendo a la necesidad de fortalecer a las instituciones empresariales en sus diferentes niveles y a la generación de mecanismos que apoyen los agrupamientos de las PYME con base en promotores que faciliten los financiamientos con garantías adecuadas" 


\section{Referencias Bibliográficas}

Ayyagari, M., Demirguc-Kunt, A. \& Vojislav, M. (2011). Small vs. Young Firms across the World. The World Bank, Development Research Group Finance and Private Sector Development Team, April 2011. Policy Research Working Paper, 5631.

Balderas, A., Gómez, J. y Allier, H. (2006). Impacto de la Apertura Comercial y la Inversión Extranjera Directa en el Crecimiento de las PYMES. Mundo Siglo XXI, 7(3) 83-87.

Banco Mundial (2013). Informe sobre el desarrollo mundial 2013. Empleo, panorama General. Washington D.C.: Banco Internacional de Reconstrucción y Fomento/Banco Mundial.

Benacek, V. (1995). Problems and environment of small businesses in the Czech Republic. Small business in the Czech Republic. (7) 437-450.

Bibu, N., Stefea, P. \& Sala, D. (2009). External and internal environment influencesiness on SME competitiveness from the western area of Romania. Scientific Review Paper, 6 (2) 41-52.

Brown, F. y Domínguez, L. (2010). Políticas en instituciones de apoyo a la pequeña y mediana empresas en México, cap. VII. En Políticas de apoyo a las PYMEs en América Latina, Ferraro y Stumpo (Comp.). Cepal: Santiago de Chile.

Carree, M., Van, Ac., Thurik, R. \& Wennekers, S. (2002). Economic Development and Business Ownership: An Analysis Using Data of 23 OECD Countries in the Period 1976-1996. Small Business Economics. 19: 271-290.

Carroll, R., Holtz-Eakin, D., Rider, M., \& Rosen, H. (2000). Personal income taxes and the growth of small firmes. National Bureau of Economic Research. Working paper 7980.

CBO (Congressional Budget Office) (2012). Small Firms, Employment, and Federal Policy. USA: Congress.

Choi, Y. \& Phan, P. (2006). Influences of Economic and Technology Policy on the Dynamics of New Firm Formation. Small Business Economics. 26: 493-503.

Cohen, M. y Baralla, G. (2012). La situación de las PYMES en América Latina. Argentina: Ireal PYME.org.

Comunidad Europea (CE) (2006). La nueva definición de PYME. Publicaciones de empresa e Industria, Comunidad Europea.

De Kok, J., Deijl, C. \& Veldhuis-Van, C. (2013). Is Small Still Beautiful? Alemania: International Labour Organization and Deutsche Gesellschaft für Zusammenarbeit (GIZ) GmbH.

Di Tomaso, M. R. y Dubbini, S. (2000). Towards a theory of the small firms: theoretical aspects an some politicy implications. Chile: Cepal.
DOF (Diario oficial de la federación). (2007) Plan nacional de desarrollo 2007-2012. México: 31 de mayo de 2007.

DOF (Diario oficial de la federación). (2008) Programa sectorial de economía 2007-2012. México: 14 de mayo de 2008.

DOF (Diario Oficial de la Federación). México: 30 de junio 2009. DOF (Diario oficial de la Federación). (2013) Acuerdo que regula la organización y funcionamiento interno del Instituto Nacional del Emprendedor. México: 15 de abril de 2013.

Dussel, E. (2004). Pequeña y mediana empresa en México: condiciones, relevancia en la economía y retos de política. Economía UNAM, 2, 64-84.

Haltiwanger, J., Jarmin, R. \& Miranda, J. (2011). Who Creates Jobs? Small vs. Large vs. Young. National Bureau of Economic Research, Working Paper 16300.

Hualde, A. (1998). PYMEs y desarrollo regional: La importancia de los enfoques europeos y sus limitaciones. Perfiles Latinoamericanos, 13, 199-227.

INEGI (2010). Censos Económicos 2009. México: Instituto Nacional de Estadística Geografía e Informática.

INEGI. Censos Económicos: 1994,1998, 2003, en www.inegi. gob.mx

Kliesen, K. y Maués, J. (2011). Are Small Businesses the Biggest Producers of Jobs? The regional economics, abril 2011, 8-9.

Leebaert, D. (2006). How Small Businesses Contribute to U.S. Economic Expansion. eJournal USA, 11 (1) 3-6.

Miliaras, C. (2012). Creating Jobs That Reduce Poverty: A Research Agenda on Developing-Country Gazelle. RTI Press publication No. 0P-0011-1211. Research Triangle Park, NC: RTI Press. Retrieved from http://www.rti.org/rtipress.

Neumark, D., Wall, B. \& Zhang, J. (2008). Do small businesses create more jobs? National Bureau of Economic Research, Working Paper 13818.

Olawale, y Garwe, (2010). Obstacles to the growth of new SMEs in South Africa: A principal component analysis approach. African Journal of Business Management, 4 (5) 729-738.

Pavón, L. (2010). Financiamiento a las Micro Empresas y las PYMEs en México (2000-2009). Chile: Naciones Unidas-CEPAL.

Ramírez, E. (2007). Crítica a la estructura tributaria actual en México. Contaduría y Administración, 223, 113-134.

Rivera, G. (Coord.) (2002). Micro, pequeñas y medianas empresas en México. Evolución, funcionamiento y problemática. México: Instituto de Investigaciones Legislativas del Senado de la República.

Roper, S. \& Hart, M. (2005). Small Firm Growth and Public Policy in the UK: what exactly are the connections? Aston Business School Research Papers. RP 0504.

Saavedra, M. y Hernández, Y. (2008). Caracterización e Importancia de las PYME en Latinoamérica: Un estudio Comparativo. Revista Actualidad Contable Faces, 11 (17), 122-134. 
La PYME como generadora de empleo en México

Saavedra, M. y Tapia, B. (2012) El Entorno Sociocultural y la Competitividad de la PYME en México. Panorama socioeconómico, 30 (44) 4-24.

Saavedra, M., Tapia, B. y Anaya, A. (2013). El impacto de las Políticas Públicas en la MIPYME mexicana. Ciencias Administrativas, 1 (1) 1-19.

Smallbone, D. y Welter, F. (2001). The role of government in SME development in the transition economies of central and Eastern and the newly independent states. Conference proceedings the fourth International Conference on
Enterprise in Transition. University of Split, Split-Hvar, May 24-26, 2001 Croacia.

Vandenberg, P. (2007). Poverty reduction through small enterprises Emerging consensus, unresolved issues and ILO activities. Small Enterprise Development Programme Job Creation and Enterprise Development Department International Labour Office - Geneva.

Zevallos, E. (2006). Obstáculos al desarrollo de las pequeñas y medianas empresas en América Latina. Cuadernos de difusión, 11 (20) 75-96. 